\title{
In-Phase and Anti-Phase Synchronization in a Laser Frequency Comb
}

\author{
Johannes Hillbrand®,$^{1,2, *}$ Dominik Auth $\odot,{ }^{3}$ Marco Piccardo $\odot,{ }^{2}$ Nikola Opačak $\odot,{ }^{1}$ Erich Gornik $\odot,{ }^{1}$ \\ Gottfried Strasser $\odot,{ }^{1}$ Federico Capasso, ${ }^{2}$ Stefan Breuer, ${ }^{3}$ and Benedikt Schwarz $\odot^{1,2, \dagger}$ \\ ${ }^{1}$ Institute of Solid State Electronics, TU Wien, Gusshausstrasse 25-25a, 1040 Vienna, Austria \\ ${ }^{2}$ John A. Paulson School of Engineering and Applied Sciences, Harvard University, Cambridge, Massachusetts 02138, USA \\ ${ }^{3}$ Institute of Applied Physics, Technische Universität Darmstadt, Schlossgartenstrasse 7, 64289 Darmstadt, Germany
}

(Received 20 September 2019; published 14 January 2020)

\begin{abstract}
Coupled clocks are a classic example of a synchronization system leading to periodic collective oscillations. Already in 1665, Christiaan Huygens described this phenomenon as a kind of "sympathy" among oscillators. In this work, we describe the formation of two types of laser frequency combs as a system of oscillators coupled through the beating of the lasing modes. We experimentally show two completely different types of synchronization in a quantum dot laser-in-phase and splay-phase states. Both states can be generated in the same device, just by varying the damping losses of the system. This modifies the coupling among the oscillators. The temporal laser output is characterized using both linear and quadratic autocorrelation techniques. Our results show that both pulses and frequency-modulated states can be generated on demand within the same device. These findings allow us to connect laser frequency combs produced by amplitude-modulated and frequency-modulated lasers and link these to pattern formation in coupled systems such as Josephson-junction arrays.
\end{abstract}

DOI: 10.1103/PhysRevLett.124.023901

The spectrum of an optical frequency comb (OFC) consists of a set of equidistant modes [1]. In the time domain, they produce a periodic intensity with a temporal waveform depending on the phase relationship between the modes. For the vast majority of laser frequency combs, such temporal waveform will be predominantly characterized by one of the two following behaviors: amplitudemodulated (AM) mode-locked lasers, in which OFCs were first demonstrated, emit a periodic train of light pulses $[2,3]$. They rely on mechanisms such as saturable absorption and Kerr lensing. On the other hand, frequencymodulated (FM) lasers, which are mostly semiconductor based [4-8] and started emerging more recently, produce a quasicontinuous-wave intensity accompanied by a frequency chirp. FM comb formation has mostly been related to ultrafast gain dynamics that lead to efficient four-wave mixing [4,9-11], while fundamental self-starting AM comb formation is known to occur in slow gain media with gain relaxation times longer than the cavity round-trip time. Hence, there is an established belief that AM and FM comb formation exclude each other.

In this work, we show that the formation of a frequency comb can also be understood as synchronization of coupled

Published by the American Physical Society under the terms of the Creative Commons Attribution 4.0 International license. Further distribution of this work must maintain attribution to the author(s) and the published article's title, journal citation, and DOI. oscillators. Such synchronization phenomena were previously observed in laser arrays and in microresonator frequency combs [12-14]. Each pair of neighboring comb lines produces a beating at their difference frequency. The collective action of these intermode beatings generates the laser beat note [Fig. 1(a)]. This beat note appears narrow when a comb is formed, indicating the equidistantly spaced comb lines. Its amplitude depends on the phase relation between the modes. In-phase synchronization leads to an AM comb with strong beat note. In contrast, antiphase synchronization generates an FM comb and suppresses the beat note. The particular antiphase state observed in FM combs $[5,7,15]$ corresponds to splay-phase synchronization, where the phases are splayed uniformly around the unit circle. This results in mutual annihilation of the intermode beatings.

This interpretation allows us to connect frequency combs to coupled clocks. The first studies of antiphase synchronization trace back to 1665, when Christiaan Huygens noticed an intriguing phenomenon: two of his newly invented pendulum clocks, mounted on a wooden bar, were swinging in perfect consonance in opposite direction [16]. He described it as "an odd kind of sympathy." His observation is illustrated using coupled metronomes in Fig. 1(b). While in this configuration the metronomes tend to synchronize in-phase, the original observation of Huygens can be reproduced by introducing a damping mechanism that suppresses the movement of the base [17]. Hence, the damping (or the dissipation) in the system decides, which of the two states will be observed. 

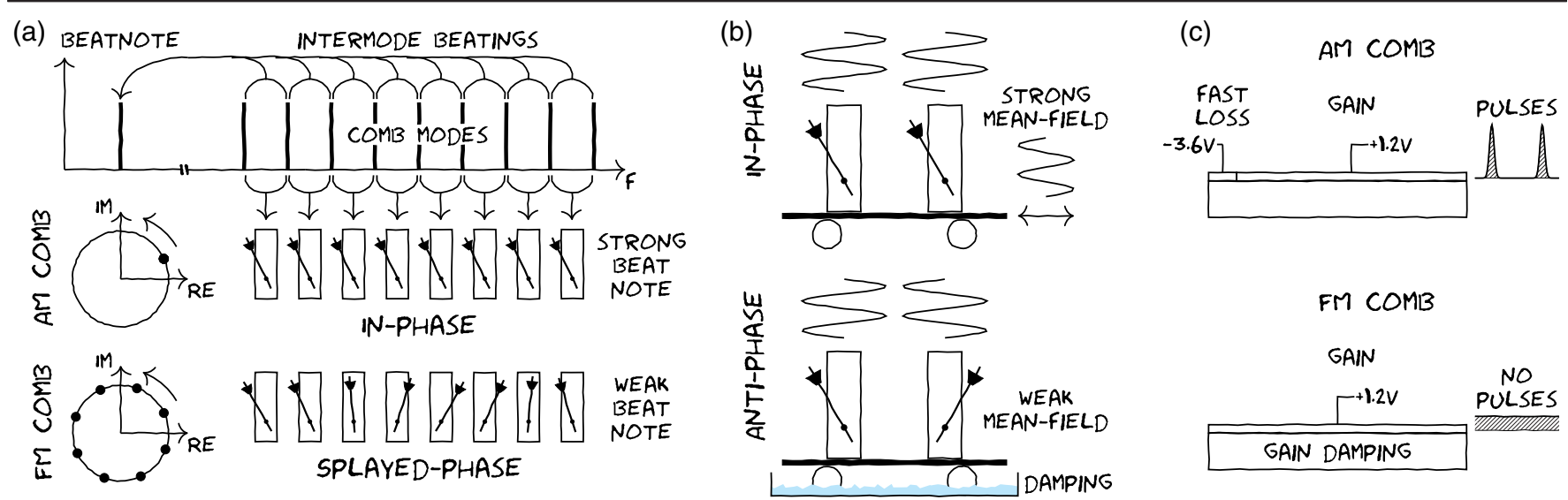

FIG. 1. (a) Different synchronization states of intermode beatings. Every beating represents an oscillator illustrated by metronomes. These oscillators are coupled globally through their collective action-the laser beat note. Depending on the coupling, two distinct synchronization states are observed. In an AM comb, they are synchronized in-phase leading to strong amplitude modulation and pulses. In an FM comb, the phases are splayed uniformly across the unit circle. Hence, intermode beatings oscillating out of phase by $\pi$ mutually annihilate each other. This suppresses amplitude modulations and the beat note. (b) The same can be observed in a system of two coupled metronomes on a movable platform. Metronomes positioned on top of the platform will synchronize either in-phase or in antiphase, depending on the damping. Such damping can be added by placing the system in a water bath. (c) Illustrating the analogy to $\mathrm{AM}$ and FM comb generation with semiconductor lasers. AM combs can be generated through passive mode locking via fast saturable loss, while FM comb can even be realized in single section lasers. There, gain saturation dampens amplitude modulations and the beat note similarly to the water bath in (b).

We show that this interpretation can be applied to semiconductor lasers. We demonstrate experimentally that both $\mathrm{AM}$ and FM comb states can be generated in the very same laser, while leaving the gain dynamics unchanged. Therefore, we can conclude that AM and FM comb generation in semiconductor lasers does not exclude each other.

Semiconductor quantum dot lasers (QDLs) constitute a unique platform for this experimental study. QDLs emit in the near-infrared region, offer low threshold current and broad spectral coverage [18-20]. Their active material is endowed with properties enabling both AM and FM combs. On one hand, the optical gain in QDLs is provided by interband transitions with an upper-state lifetime much longer than the cavity round-trip time. Then, by applying a reverse bias to a short section, the active region becomes a fast saturable absorber [18,21]. This allows AM comb operation by passive mode locking [22-25] [Fig. 1(c) top]. On the other hand, carrier diffusion is strongly suppressed due to carrier localization in the quantum dots [26]. Hence, the standing-wave pattern formed by the longitudinal cavity modes of a Fabry-Perot QDL leads to spatial hole burning. This triggers the multimode instability required for FM combs $[27,28]$. FM combs have mostly been observed in single section QDLs and have been referred to as self-mode locking [6,8,29] [Fig. 1(c) bottom].

The investigated laser is a $4 \mathrm{~mm}$ long monolithic InAs/InGaAs QDL emitting at around $1265 \mathrm{~nm}$ $\left(7900 \mathrm{~cm}^{-1}\right)$. The electrical contacts are divided into two gain sections and two $200 \mu \mathrm{m}$ long absorber sections [Fig. 2(a)]. When all sections are biased homogeneously, the lasing threshold is at $45 \mathrm{~mA}$ with a slope efficiency of $0.22 \mathrm{~W} / \mathrm{A}$. In the following experiments, the bias of the gain section is kept constant at $160 \mathrm{~mA}$ to ensure that the gain dynamics remain unchanged. This allows us to prove that AM and FM comb generation does not exclude each other in terms of gain dynamics. The absorber bias was tuned to identify regions with narrow beat note. Figure 2(b) shows two beat notes obtained with a fast photodiode and for two selected absorber voltages of $0 \mathrm{~V}$ (red) and $-3.8 \mathrm{~V}$ (blue). At $-3.8 \mathrm{~V}$ reverse bias, the photogenerated carriers can escape the quantum dots efficiently, resulting in fast saturable losses. There, the laser operates in the AM regime, exhibiting a narrow and strong beat note caused by passive mode locking with fast saturable absorption. The intensity autocorrelation [Fig. 2(c)] proves that optical pulses are emitted in this regime. We retrieve an optical pulse width of $10 \mathrm{ps}$ from the intensity autocorrelation width of 14 ps (FWHM) by assuming a Gaussian shape.

The laser dynamics change drastically, when the fast saturable absorption is switched off by turning off the reverse bias. In these conditions, the laser no longer generates optical pulses because the gain medium damps amplitude modulations. This is because an amplitudemodulated intensity saturates the gain stronger than its continuous average. Hence, a continuous waveform experiences more gain than an AM waveform. Surprisingly, still a narrow beat note is observed. This indicates that the QDL is still operating as frequency comb without fast saturable losses. The suppression of amplitude modulations is evident in the strong attenuation of the beat note by $31 \mathrm{~dB}$ [Fig. 2(b)]. The reason for this is that by switching 
(a)

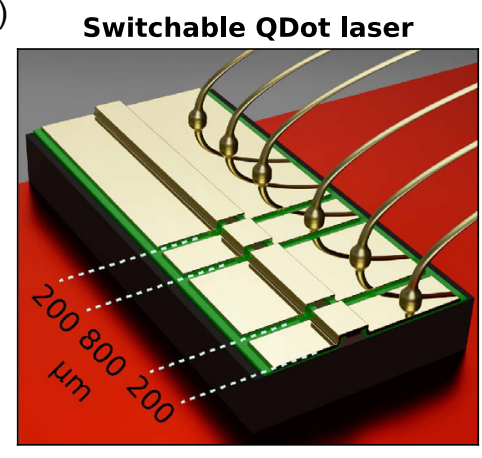

(b)

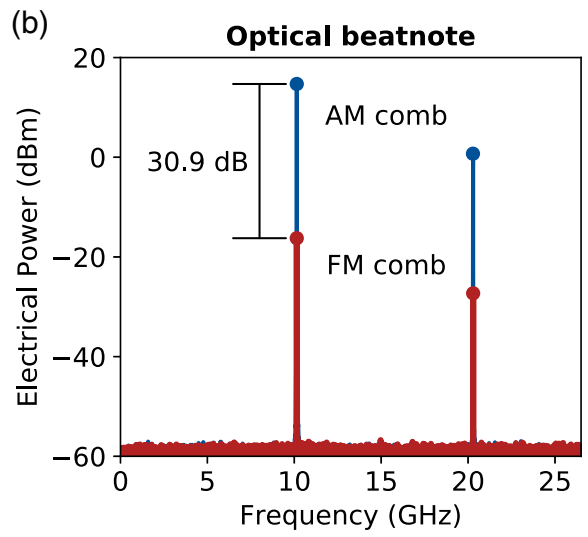

(c)

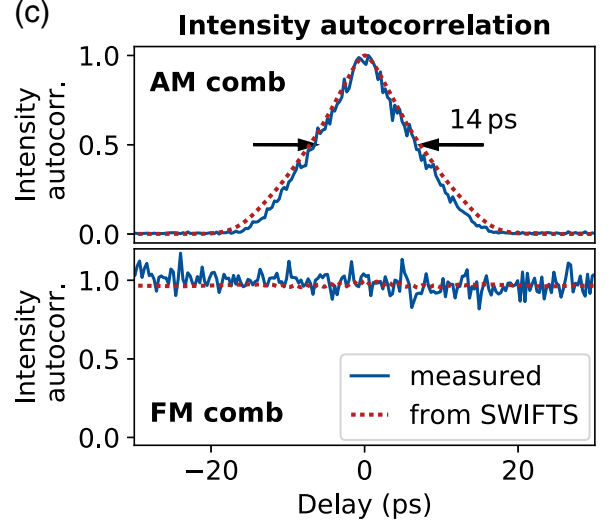

FIG. 2. (a) Schematic of the investigated $4 \mathrm{~mm}$ long QDL with two gain sections and two $200 \mu \mathrm{m}$ long absorber sections. (b) First and second harmonic beat notes of the QDL operating in the AM (blue) and FM (red) regimes. The weaker beat note of the FM comb indicates the strong suppression of amplitude modulations in the FM regime. The beat note was measured using a fast photodiode. (c) Characterization of the AM and FM states by intensity autocorrelation measurements (blue lines). In the AM regime, the autocorrelation shows a FWHM of $14 \mathrm{ps}$, corresponding to $10 \mathrm{ps}$ pulse width assuming a Gaussian shape. The red dotted line corresponds to the calculated autocorrelation using the SWIFTS time traces shown in Figs. 3(a) and 3(b), highlighting the good qualitative agreement between both experimental techniques.

off the fast saturable loss, the laser switches from AM to FM comb operation. The corresponding intensity autocorrelation in Fig. 2(c) confirms the expected flat and quasicontinuouswave laser output, connected to the suppressed beat note. However, it also shows that intensity autocorrelation yields useful information only for AM combs.

Shifted-wave interference Fourier transform spectroscopy (SWIFTS) is an ideal measurement technique to study both AM and FM combs [30]. SWIFTS enables the measurement of the phase difference of adjacent modes. This knowledge provides full insight into the comb operation, enabling direct reconstruction of intensity and instantaneous frequency. Details can be found in Ref. [30] and in Fig. S1 of the Supplemental Material [31]. Figure 3(a) shows the SWIFTS results with reverse biased absorber at $-3.8 \mathrm{~V}$. The spectrum of the QDL spans across $30 \mathrm{~cm}^{-1}$ and consists of a group of modes centered at $7908 \mathrm{~cm}^{-1}$ and a tail toward lower wave numbers. The full SWIFTS characterization is shown in Fig. S2 of the Supplemental Material [31]. The intermode beating phases $\Delta \phi$ are mostly synchronized inphase with a small remaining chirp at the high-energy end of the spectrum. Consequently, they span across a narrow range of $0.43 \pi . \Delta \phi$ is related to the spectral group delay with $2 \pi$ corresponding to exactly one cavity round-trip period. Hence, we expect nontransformation-limited optical pulses with $0.43 \pi / 2 \pi=21.5 \%$ duty cycle.

Indeed, the reconstructed time signal depicted in Fig. 3(b) confirms pulses with 8.9 ps full width at half maximum (FWHM). The peak power is $167 \mathrm{~mW}$ corresponding to an enhancement of 10.7 with respect to the average power of $15.6 \mathrm{~mW}$. The pulses show an intense initial burst followed by a trailing edge. In order to independently verify the SWIFTS results, we show the excellent agreement of the time-domain reconstruction retrieved from SWIFTS with the intensity autocorrelation in Fig. 2(c). The instantaneous wave number [Fig. 3(b)] allows us to identify the spectral regions responsible for this pulse shape. While the intense initial burst contains the wave numbers up to $7908 \mathrm{~cm}^{-1}$ [tail of the spectrum in Fig. 3(a)], the trailing edge of the pulse is caused by wave numbers above $7908 \mathrm{~cm}^{-1}$.

The SWIFTS characterization of the FM state is shown in Fig. 3(c). In contrast to the AM comb state, the intermode difference phases are uniformly distributed over the range of $2 \pi$. This linear phase pattern corresponds to splay-phase synchronization. The corresponding time domain signal [Fig. 3(c) top] shows almost no amplitude modulation and a linearly chirped instantaneous frequency. This is in good agreement with previous experimental observations of a strong chirp in QDL [6] and explains the flat intensity autocorrelation in Fig. 2(c). The same phenomenon was observed in other lasers, such as quantum and interband cascade lasers $[5,15,32]$. The splayed phases, covering exactly the range of $2 \pi$, were found in all experiments on these devices regardless if the laser was free running or electrically injection locked [32].

Figure 3(e) shows the results of numerical simulations based on the time-domain traveling-wave model presented in Ref. [28], which was extended to slow gain media. In particular, we use a gain relaxation time of $1 \mathrm{~ns}$ with an additional fast contribution of $10 \%$. This fast contribution, which is known from experiments [18], is necessary for the stability of the model. A more detailed description is provided in the Supplemental Material [31]. The experimental time traces for the QDL output power and instantaneous frequency [Figs. 3(b) and 3(d)] are recreated. In the FM state, where the device is uniformly biased [Fig. 3(e) right], a quasicontinuous-wave intensity is 


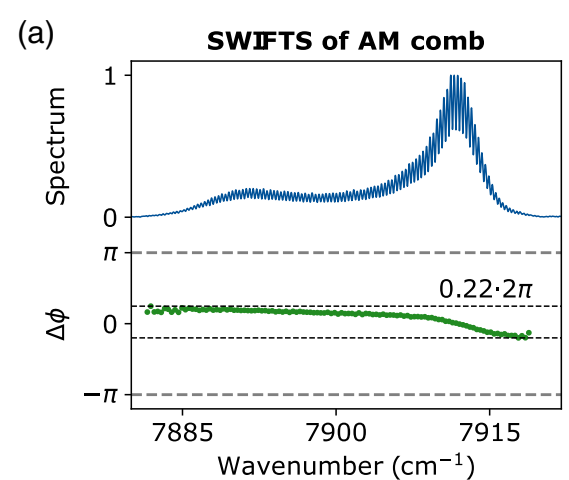

(b)

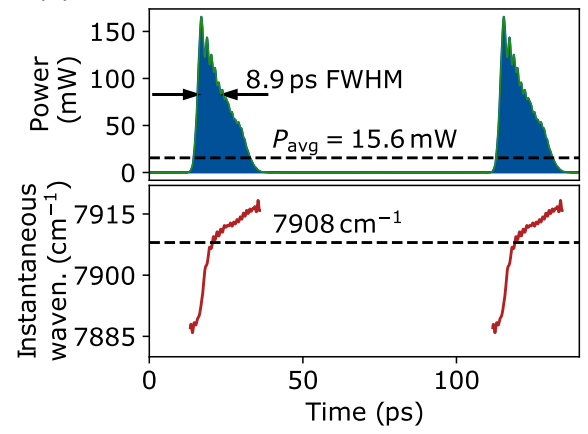

(c)

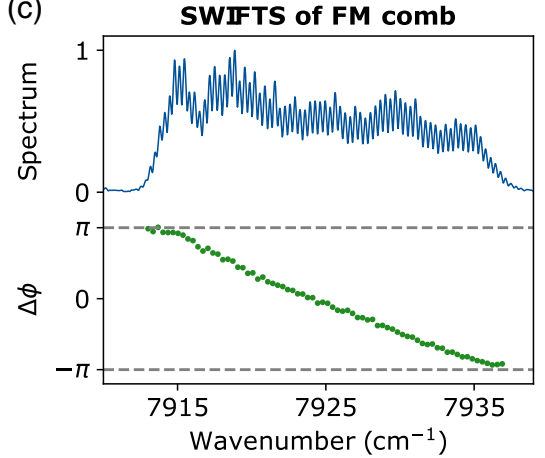

(d)

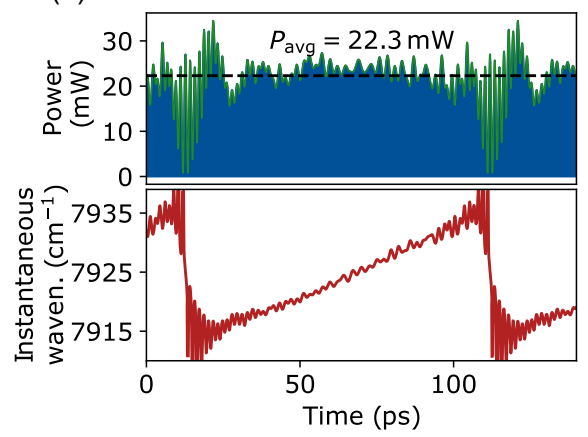

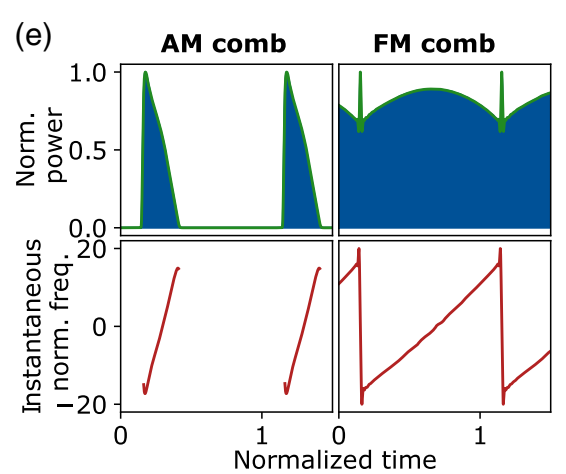

(f)

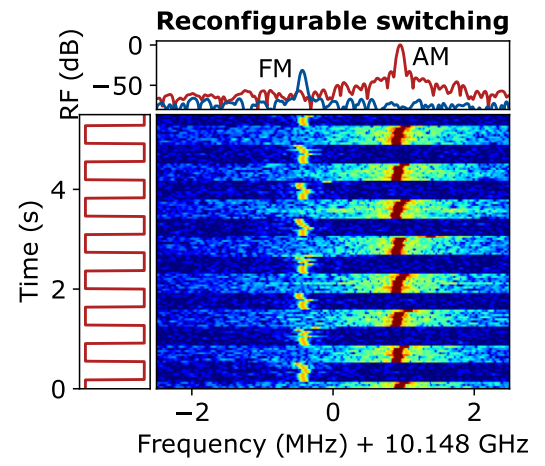

FIG. 3. (a) SWIFTS characterization of the AM comb. The spectrum (blue) spans over roughly $30 \mathrm{~cm}^{-1}$. The intermodal difference phases $\Delta \phi$ (green dots) cover a range of $0.43 \pi$. (b) The reconstructed optical power yields optical pulses with 8.9 ps FWHM. The instantaneous frequency allows us to identify the spectral region responsible for the trailing edge of the pulses. (c) SWIFTS characterization of the FM comb. $\Delta \phi$ are now splayed across the full range of $2 \pi$. (d) The reconstructed optical power shows almost no amplitude modulation while the instantaneous frequency is linearly chirped across one round-trip period. (e) Numerical simulations results, depicting the normalized power and instantaneous frequency in the case of both AM and FM combs. The instantaneous frequency and time are normalized to the cavity round-trip time. (f) Color-coded radio frequency (rf) spectrum of the QDL while a rectangular modulation between 0 and $-3.8 \mathrm{~V}$ is applied to the absorber sections, while the gain section remains unchanged.

obtained due to gain dampening of amplitude modulations. The simulated instantaneous frequency shows the same linear chirp as observed experimentally [Fig. 3(d)]. This phenomenon is caused by the interplay between group velocity dispersion (GVD) and Kerr nonlinearity [28]. As the GVD contribution is independent from the time dynamics of the gain medium, it provides further evidence that FM combs are not limited to ultrafast gain dynamics. On the other hand, we model passive mode locking by applying a reverse bias, lowering the saturation intensity and absorption recovery time in a short section. As a result, chirped pulses exhibiting a similar trailing edge as in the experiment are obtained [Fig. 3(e) left].

The damping mechanism choosing the comb state is due to the gain medium itself. Switching between AM and FM states is here performed by introducing the reversed effect through saturable losses. In order to show that switching can be achieved temporally reconfigurably, we apply a periodic rectangular voltage modulation to the absorber section between 0 and $-3.8 \mathrm{~V}$. The temporal evolution of the beat note is plotted in Fig. 3(f). Indeed, the QDL switches back and forth between AM and FM states by periodically changing the damping mechanism.
In conclusion, we demonstrated AM and FM comb operation-two distinct frequency comb states with entirely different temporal dynamics - in a single semiconductor quantum dot laser by changing the absorber bias. Their underlying physical mechanisms can be directly related to oscillators coupled through the intermode beating. By both nonlinear intensity autocorrelation and SWIFTS, we identified optical pulse shape and width as well as the specific spectral regions responsible for pulse broadening. We numerically and experimentally highlighted that the requirements on the gain dynamics for $\mathrm{AM}$ and FM combs do not necessarily exclude each other. FM combs can be generated also with slower gain media, where most of the gain relaxes slower than the round-trip time, spatial hole burning is strong enough to trigger multimode operation, and sufficient group velocity dispersion is present.

This work was supported by the Austrian Science Fund (FWF) within the projects NanoPlas (P28914-N27) and "Building Solids for Function" (Project No. W1243), the city of Vienna within the "Hochschuljubiläumsstiftung," the German Research Foundation (DFG) (Grant No. 389193326), and the Adolf Messer Foundation 
(doctoral fellowship). M. P. and F. C. acknowledge support from the National Science Foundation under Grant No. CCSS-1807323. Any opinions, findings, conclusions, or recommendations expressed in this material are those of the authors and do not necessarily reflect the views of the National Science Foundation.

*johannes.hillbrand@tuwien.ac.at †benedikt.schwarz@tuwien.ac.at

[1] T. Udem, R. Holzwarth, and T. W. Hänsch, Optical frequency metrology, Nature (London) 416, 233 (2002).

[2] F. Keilmann, C. Gohle, and R. Holzwarth, Time-domain mid-infrared frequency-comb spectrometer, Opt. Lett. 29, 1542 (2004).

[3] Y. Silberberg, D. A. B. Miller, A. C. Gossard, W. Wiegmann, P. W. Smith, and D. J. Eilenberger, Passive mode locking of a semiconductor diode laser, Opt. Lett. 9, 507 (1984).

[4] A. Hugi, G. Villares, S. Blaser, H. C. Liu, and J. Faist, Mid-infrared frequency comb based on a quantum cascade laser, Nature (London) 492, 229 (2012).

[5] B. Schwarz, J. Hillbrand, M. Beiser, A. M. Andrews, G. Strasser, H. Detz, A. Schade, R. Weih, and S. Höfling, Monolithic frequency comb platform based on interband cascade lasers and detectors, Optica 6, 890 (2019).

[6] M. J. R. Heck, E. A. J. M. Bente, B. Smalbrugge, Y.-S. Oei, M. K. Smit, S. Anantathanasarn, and R. Nötzel, Observation of q-switching and mode-locking in two-section InAs/InP (100) quantum dot lasers around $1.55 \mu \mathrm{m}$, Opt. Express 15, 16292 (2007).

[7] R. Rosales, S. G. Murdoch, R. Watts, K. Merghem, A. Martinez, F. Lelarge, A. Accard, L. P. Barry, and A. Ramdane, High performance mode locking characteristics of single section quantum dash lasers, Opt. Express 20, 8649 (2012).

[8] C. Weber, L. L. Columbo, M. Gioannini, S. Breuer, and P. Bardella, Threshold behavior of optical frequency comb self-generation in an InAs/InGaAs quantum dot laser, Opt. Lett. 44, 3478 (2019).

[9] W. Kuehn, W. Parz, P. Gaal, K. Reimann, M. Woerner, T. Elsaesser, T. Müller, J. Darmo, K. Unterrainer, M. Austerer, G. Strasser, L. R. Wilson, J. W. Cockburn, A. B. Krysa, and J.S. Roberts, Ultrafast phase-resolved pump-probe measurements on a quantum cascade laser, Appl. Phys. Lett. 93, 151106 (2008).

[10] P. Borri, S. Schneider, W. Langbein, and D. Bimberg, Ultrafast carrier dynamics in In-GaAs quantum dot materials and devices, J. Opt. A 8, S33 (2006).

[11] P. Bardella, L. L. Columbo, and M. Gioannini, Selfgeneration of optical frequency comb in single section quantum dot Fabry-Perot lasers: a theoretical study, Opt. Express 25, 26234 (2017).

[12] Y. H. Wen, M. R. E. Lamont, S. H. Strogatz, and A. L. Gaeta, Self-organization in kerr-cavity-soliton formation in parametric frequency combs, Phys. Rev. A 94, 063843 (2016).

[13] P. Del'Haye, A. Coillet, W. Loh, K. Beha, S. B. Papp, and S. A. Diddams, Phase steps and resonator detuning measurements in microresonator frequency combs, Nat. Commun. 6, 5668 (2015).

[14] P. Del'Haye, K. Beha, S. B. Papp, and S. A. Diddams, Selfinjection locking and phaselocked states in microresonatorbased optical frequency combs, Phys. Rev. Lett. 112, 043905 (2014).

[15] M. Singleton, P. Jouy, M. Beck, and J. Faist, Evidence of linear chirp in mid-infrared quantum cascade lasers, Optica 5, 948 (2018).

[16] C. Huygens, Oeuvres complètes de Christiaan Huygens. Publiées par la Société Hollandaise des Sciences (La Haye, M. Nijhoff, 1888).

[17] J. Pantaleone, Synchronization of metronomes, Am. J. Phys. 70, 992 (2002).

[18] E. U. Rafailov, M. A. Cataluna, and W. Sibbett, Modelocked quantum-dot lasers, Nat. Photonics 1, 395 (2007).

[19] H. Liu, D. Childs, T. Badcock, K. Groom, I. Sellers, M. Hopkinson, R. Hogg, D. Robbins, D. Mowbray, and M. Skolnick, High-performance threelayer $1.3 \mu \mathrm{m}$ InAs-GaAs quantum-dot lasers with very low continuous-wave roomtemperature threshold currents, IEEE Photonics Technol. Lett. 17, 1139 (2005).

[20] D. Bimberg, N. Kirstaedter, N. N. Ledentsov, Z. I. Alferov, P. S. Kop'ev, and V. M. Ustinov, InGaAs-GaAs quantumdot lasers, IEEE J. Sel. Top. Quantum Electron. 3, 196 (1997).

[21] M. G. Thompson, A. Rae, R. L. Sellin, C. Marinelli, R. V. Penty, I. H. White, A. R. Kovsh, S. S. Mikhrin, D. A. Livshits, and I. L. Krestnikov, Subpicosecond high-power mode locking using ared waveguide monolithic quantumdot lasers, Appl. Phys. Lett. 88, 133119 (2006).

[22] H. Haus, Mode-locking of lasers, IEEE J. Sel. Top. Quantum Electron. 6, 1173 (2000).

[23] P. Bardella, L. Drzewietzki, M. Krakowski, I. Krestnikov, and S. Breuer, Mode locking in a tapered two-section quantum dot laser: design and experiment, Opt. Lett. 43, 2827 (2018).

[24] S. Meinecke, L. Drzewietzki, C. Weber, B. Lingnau, S. Breuer, and K. Ldge, Ultra-short pulse generation in a three section tapered passively mode-locked quantum-dot semiconductor laser, Sci. Rep. 9, 1783 (2019).

[25] Y.-C. Xin, Y. Li, V. Kovanis, A. L. Gray, L. Zhang, and L. F. Lester, Recongurable quantum dot monolithic multisection passive mode-locked lasers, Opt. Express 15, 7623 (2007).

[26] P. Bardella, L. L. Columbo, and M. Gioannini, Selfgeneration of optical frequency comb in single section quantum dot fabry-perot lasers: a theoretical study, Opt. Express 25, 26234 (2017).

[27] C. L. Tang, H. Statz, and G. deMars, Spectral output and spiking behavior of solid-state lasers, J. Appl. Phys. 34, 2289 (1963).

[28] N. Opačak and B. Schwarz, Theory of frequencymodulated combs in lasers with spatial hole burning, dispersion, and kerr nonlinearity, Phys. Rev. Lett. 123, 243902 (2019).

[29] R. Rosales, K. Merghem, C. Calo, G. Bouwmans, I. Krestnikov, A. Martinez, and A. Ramdane, Optical pulse generation in single section InAs/GaAs quantum dot edge 
emitting lasers under continuous wave operation, Appl. Phys. Lett. 101, 221113 (2012).

[30] D. Burghoff, Y. Yang, D. J. Hayton, J.-R. Gao, J. L. Reno, and Q. Hu, Evaluating the coherence and time-domain prole of quantum cascade laser frequency combs, Opt. Express 23, 1190 (2015).

[31] See Supplemental Material at http://link.aps.org/ supplemental/10.1103/PhysRevLett.124.023901 for a detailed description of the shifted wave interference Fourier transform spectroscopy technique, as well as the theoretical model used for the numerical simulations.

[32] J. Hillbrand, A. M. Andrews, H. Detz, G. Strasser, and B. Schwarz, Coherent injection locking of quantum cascade laser frequency combs, Nat. Photonics 13, 101 (2019). 